\title{
REPRESENTATIVES AND COMMITTEES OF THE SOCIETY
}

Representatives of the Society in the Division of Physical Sciences of the National Research Council:

1938-1939-T. C. Fry, Solomon Lefschetz, J. F. Ritt.

1939-1940-Solomon Lefschetz, J. F. Ritt, Oystein Ore.

1940-1941-J. F. Ritt, Oystein Ore, G. C. Evans.

Representatives on the Council of the American Association for the Advancement of Science:

1939-L. L. Dines, R. E. Langer.

1940-Arnold Dresden, J. R. Kline.

Representatives on the Editorial Board of the Annals of Mathematics:

T. H. Hildebrandt, Saunders MacLane, G. T. Whyburn.

Representatives on the Editorial Board of the Duke Mathematical Journal:

Oystein Ore, G. T. Whyburn.

Representative on the American Year Book:

C. N. Moore.

Representative on the Sectional Committee on Standards for Graphical Presentation sponsored by the American Society of Mechanical Engineers: W. H. Roever.

Representative on the Joint Committee on Engineering Statistics sponsored by the American Society of Mechanical Engineers and the American Society for Testing Materials:

A. A. Bennett.

Representative on the Joint Committee for the Development of Statistical Applications in Engineering and Manufacturing:

E. V. Huntington.

Colloquium Speakers:

1939-A. A. Albert, M. H. Stone. 1941-Oystein Ore.

1940-G. T. Whyburn. 1942-R. L. Wilder.

Committee on Gibbs Lecturers:

A. B. Coble, G. C. Evans, M. H. Stone.

Gibbs Lecturers:

1923-M. I. Pupin.

1924-Robert Henderson.

1925-James Pierpont.

1926-H. B. Williams.

1927-E. W. Brown.
1928-G. H. Hardy.

1929-Irving Fisher.

1936-H. N. Russell.

1931-P. W. Bridgman. 1937-C. A. Kraus.

1932-R. C. Tolman. 1939-Theodore von Kármán.

Committee on Society Visiting Lectureship:

G. D. Birkhoff, G. A. Bliss, E. R. Hedrick. 


\section{Visiting Lecturers:}

1927-1928-Constantin Carathéodory, University of Munich.

1928-1929-Hermann Weyl, Zurich Technical School.

1929-1930-Enrico Bompiani, University of Rome.

1930-1931-Wilhelm Blaschke, University of Hamburg.

1931-1932-R. L. Moore, University of Texas.

1936-1937-Thirukkannapuram Vijayaraghavan, University of Dacca.

Committee on the Moore Fund:

T. H. Hildebrandt, R. D. Carmichael, Arnold Dresden.

Committee on Publication of Moore Manuscript:

R. W. Barnard, E. W. Chittenden, L. M. Graves, T. H. Hildebrandt, M. H Ingraham.

\section{Committee on Printing Contracts:}

D. R. Curtiss, M. H. Ingraham, C. C. MacDuffee.

\section{Committee on Publicity:}

R. M. Foster, Saunders MacLane, G. B. Price, J. B. Rosser, J. M. Thompson.

\section{Committees to Select Hour Speakers:}

For Annual and Summer Meetings, R. G. D. Richardson, L. M. Graves, J. R. Kline.

For Eastern Sectional Meetings, T. R. Hollcroft, Einar Hille, J. L. Walsh.

For Western Sectional Meetings, W. L. Ayres, G. A. Bliss, E. W. Chittenden.

For Far Western Sectional Meetings, T. M. Putnam, E. R. Hedrick, Gabor Szegö.

Emergency Executive Committee of the International Congress of Mathematicians :

G. D. Birkhoff, W. C. Graustein, Einar Hille, M. H. Ingraham, J. R. Kline, Marston Morse, R. G. D. Richardson, M. H. Stone.

Executive Committee of Mathematical Reviews:

Oswald Veblen, T. C. Fry.

Publicity Committee for Mathematical Reviews:

G. B. Price, Virgil Snyder.

\section{FORMER PRESIDENTS}

J. H. Van Amringe, 1889-1890.

Emory McClintock, 1891-1894.

G. W. Hill, 1895-1896.

Simon Newcomb, 1897-1898.

R. S. Woodward, 1899-1900.

E. H. Moore, 1901-1902.

T. S. Fiske, 1903-1904.

W. F. Osgood, 1905-1906.

H. S. White, 1907-1908.

Maxime Bôcher, 1909-1910.

H. B. Fine, 1911-1912.

E. B. Van Vleck, 1913-1914.
E. W. Brown, 1915-1916.

L. E. Dickson, 1917-1918.

Frank Morley, 1919-1920.

G. A. Bliss, 1921-1922.

Oswald Veblen, 1923-1924.

G. D. Birkhoff, 1925-1926.

Virgil Snyder, 1927-1928.

E. R. Hedrick, 1929-1930.

L. P. Eisenhart, 1931-1932.

A. B. Coble, 1933-1934.

Solomon Lefschetz, 1935-1936.

R. L. Moore, 1937-1938. 